3

5

\title{
Application of Supercritical Carbon Dioxide in Catalyzation
} and Ni-P Electroless Plating of Nylon 6,6 Textile

${ }^{1}$ Institute of Innovative Research, Tokyo Institute of Technology, 4259 Nagatsuta, Midori-ku, Yokohama 226-8503, Japan

${ }^{2}$ CREST, Japan Science and Technology Agency, 4259 Nagatsuta, Midori-ku, Yokohama 226-8503, Japan

${ }^{3}$ Department of Clothing Environmental Science, Nara Women's University, Kita-Uoya Nishimachi, Nara-shi, Nara 630-8506, Japan

\author{
Mitsuo Sano ${ }^{1,2}$, Yuma Tahara ${ }^{3}$, Chun-Yi Chen ${ }^{1,2}$, Tso-Fu Mark Chang ${ }^{1,2}$, \\ Tomoko Hashimoto $^{3}$, Hiromichi Kurosu ${ }^{3}$, Tatsuo Sato ${ }^{1}$ and Masato Sone ${ }^{1,2} *$
}




\section{Abstract}

In this study, an electroless deposition method including catalyzation in supercritical carbon dioxide (sc- $\left.\mathrm{CO}_{2}\right)$ and plating in sc- $\mathrm{CO}_{2}$ emulsified electrolyte was applied in metallization of Nylon 6,6 textile. In order to demonstrate the effects of the sc- $-\mathrm{CO}_{2}$, the catalyzation was conducted either by the conventional method or the supercritical $\mathrm{CO}_{2}$ catalyzation (SCC) method with bis (2,4-pentandionato)-palladium. After the catalyzation process, either the conventional electroless plating or the electroless plating with supercritical $\mathrm{CO}_{2}$ emulsified electrolyte (ELP-SCE) was performed. Surface of the Ni-P coatings on the Nylon 6,6 was observed by an optical microscope and a scanning electron microscope. The Ni-P coating by the conventional catalyzation and the conventional electroless plating showed some cracks, nodules and rough structure. The Ni-P coating by the SCC and the conventional electroless plating showed smooth Ni-P coating on the surface of each fibers and had some peeled-off parts. On the other hand, uniform Ni-P coating on the surface of each fibers was obtained by the SCC and the ELP-SCE. These results demonstrated that the SCC is effective to impregnate Pd catalysts into the fibers, and the ELP-SCE can inhibit the nodule growth of the Ni-P. Thus, the proposed method can metallize the surface of each Nylon 6,6 fibers and has potential to give some functions on textiles.

Keywords: electroless plating, supercritical carbon dioxide, Ni-P, textile, metallization 


\section{Introduction}

Wearable device is a technology integrating advanced electronic devices with the flexibility of clothes that has various applications [1-3]. For example, a wearable sensor connected to the engine of a car can be used to detect alcohol level in the human body to

47 prevent drinking and driving. Sportswear utilizing a wearable sensor can have 48 significant contribution to the sports science. Textiles usually have low electrical 49 conductivity. Making the textiles electronical conductive is one of the challenging 50 points for development of the wearable devices. The electrical conductivity can be 51 improved by depositing an electrical conductive metal coating on the textile. The metal 52 coating can be deposited by sputtering [4] and chemical vapor deposition [5]. Among 53 the deposition methods, electroless plating is the most promising method in the 54 industrial level because of the simple operation and low cost process [6]. Therefore, studies on metallization of textile fiber by electroless deposition have attracted much attention. However, there are still some problems needed to be solved in the

57 conventional electroless plating (Conv-ELP). First, a pretreatment process involving usage of toxic substances such as chromate and permanganate is usually needed. The

59 toxic chemicals used in the pretreatment raises concerns in further applications of the fibers in clothing. Second, the strong acid used in the pretreatment could damage the 
61 fibers and leads to concerns in reliability of the fibers. Third, adherence of the metal

62 coating on the fiber is sometime too low for practical applications.

63 Supercritical carbon dioxide $\left(\mathrm{sc}-\mathrm{CO}_{2}\right)$ is non-polar, and the physical properties can be

64 easily tuned between a gas and a liquid. Therefore, $\mathrm{CO}_{2}$ is used as non-toxic solvents in extractions [7], separations [8,9], chemical reactions [10], and various other applications.

Recently, several researchers have reported deposition technologies using sc- $\mathrm{CO}_{2}$, such as supercritical fluid deposition [11-13] and copper electroplating with sc- $\mathrm{CO}_{2}$ using organo copper complex as the source of copper [14]. In previous studies, metallization of polymeric substrates utilizing sc- $\mathrm{CO}_{2}$ has been demonstrated $[15,16]$. The electroless plating method involves sc- $\mathrm{CO}_{2}$ catalyzation (SCC) with organo Pd complex and electroless plating in sc- $\mathrm{CO}_{2}$ emulsified electrolyte (ELP-SCE). Both the SCC and the ELP-SCE are found to be effective in improving coverage and adherence of the Ni coatings on the Kapton ${ }^{\circledR}$ polyimide films.

The successes obtained from metallization of polymeric substrates demonstrate the potential to apply the SCC and the ELP-SCE in metallization of Nylon 6,6 textile to solve the problems of the toxic chemicals used in the pretreatment and improve adhesion of the Ni-P coating on the textile. In this paper, Nylon 6,6 textile was selected as the substrate material, because of the potentials to be applied in various fields [17]. 
79 Nylon 6,6 has high biocompatibility, good resistance to hydrocarbons, high crystalline

80 melting point, and high strength [18].

81 The purposes of this paper are to solve the problems as mentioned in the previous

82 sections by utilizing the SCC and the ELP-SCE to metallize Nylon 6,6 textile that

83 would possess adequate electrical conductivity for application in wearable devices.

\section{Materials and methods}

\subsection{Materials and sample preparation} In this study, $\mathrm{CO}_{2}$ having purity of $99.99 \%$ was purchased from Nippon Tansan Co., Ltd. A $\mathrm{PdCl}_{2} / \mathrm{SnCl}_{2}$ mixture solution consisted of $\mathrm{PdCl}_{2}(100 \mathrm{mg} / \mathrm{l}), \mathrm{SnCl}_{2}(10 \mathrm{mg} / \mathrm{l})$ and $\mathrm{HCl}(87.5 \mathrm{~g} / \mathrm{l})$ was used for the conventional catalyzation (Conv-Cat). The SCC was conducted with bis (2,4-pentandionato)-palladium. A nonionic surfactant,

91 polyoxyethylene lauryl ether $\left.\left(\mathrm{C}_{12} \mathrm{H}_{25}\left(\mathrm{OCH}_{2} \mathrm{CH}_{2}\right)_{15}\right) \mathrm{OH}\right)$, purchased from Toshi Yuka

92 Co. Ltd, was used to form the emulsions composed of the Ni-P electroless plating

93 solution and $\mathrm{sc}-\mathrm{CO}_{2}$. The Ni-P electroless plating solution was commercially available

94 electrolyte purchased from Okuno Chemical Industries Co., Ltd., and the chemical 95 composition was nickel chloride (9 wt.\%), sodium hypophosphite (12 wt.\%), 
96 complexing agent (12 wt.\%), and ion-exchanged water (67 wt.\%). The textile used in

97 this study was made from Nylon 6,6 fibers.

98

99

\subsection{Experimental apparatus}

100

The high pressure apparatus (Japan Spectra Company) used in this investigation is

101 shown in Fig. 1. The reaction chamber was made of PEEK-lined stainless steel 316

102

vessel with an inner volume of $50 \mathrm{ml}$. The reaction chamber was placed in a

103 temperature-controlled air bath to control the experimental temperature. The pressure

104 inside the reaction chamber was controlled by a back pressure regulator. The Nylon 6,6

105 textile was hooked to a plastic-coated platinum wire in the reaction chamber to conduct

106 the SCC and the ELP-SCE.

107

108

\subsection{Catalyzation process}

109

No pretreatment of the Nylon 6,6 textile was conducted in this study. Two types of

110 the catalyzation were performed. The catalyzation was performed either by the

111 Conv-Cat or the SCC. The SCC was performed with $2 \mathrm{~g} / \mathrm{l}$ of bis 
112 (2,4-pentandionato)-palladium. The temperature for both the Conv-Cat and the SCC

113 was $353 \mathrm{~K}$, and the pressure for the SCC was $15 \mathrm{MPa}$. The catalyzation for the

114 Conv-Cat was 10 s. Three values of catalyzation time for the SCC were used, which

115 were 20,60 , and $120 \mathrm{~min}$.

116

117

\subsection{Electroless Plating}

118

After the catalyzation process, the electroless plating was conducted, which was

119 either the Conv-ELP or the ELP-SCE. For the Conv-ELP, the catalyzed Nylon 6,6

120 textile was immersed in $100 \%$ of the Ni-P electroless plating solution. For the

121 ELP-SCE, the catalyzed textile was immersed in the sc- $\mathrm{CO}_{2}$ emulsified bath containing

0.2 vol. $\%$ of the surfactant and $40 \mathrm{ml}$ of the Ni-P electroless plating solution. Total

123 volume of the reaction cell is $50 \mathrm{ml}$, which gave $10 \mathrm{ml}$ available for the $\mathrm{CO}_{2}$ or 20

124 vol.\% in volume fraction. Agitation of $400 \mathrm{rpm}$ was used to ensure stability of the

125 emulsions. The reaction temperature was $353 \mathrm{~K}$ for both plating method, and pressure

126 of $15 \mathrm{MPa}$ was used for the ELP-SCE. The reaction time for the Conv-ELP was 20 min.

127 Three values of the reaction time for the ELP-SCE were used, which were 20, 40, and

$12860 \mathrm{~min}$. 


\subsection{Analysis}

Surface of the Ni-P coated textile was characterized by an optical microscope (OM,

KEYENCE: VHV-5000), a scanning electron microscope (SEM, JEOL: JSM-7500) and energy dispersive X-ray spectrometry (EDS, HORIBA: EMAX Evolution) equipped in the SEM to confirm conditions of the coating and measure film thickness of the Ni-P coating on the fiber.

\section{Results and discussion}

\subsection{Surface morphology}

For metallization of the non-electrical-conductive polymers, a catalyzation process

is usually needed before the electroless plating process. Fig. 2 shows 3D images of overview of the textiles before and after the catalyzation processes. Generally, after the catalyzation process, for both the Conv-Cat and SCC, no obvious difference on the 

coverage and adherence of the metal coatings on the polymers. However, the fibers are the acid used in the $\mathrm{PdCl}_{2} / \mathrm{SnCl}_{2}$ solution [19]. The white particulates are suggested to be the damaged polymers stayed on the fibers after the Conv-Cat step, shown in Fig. 3 (d). In general, the rough surface is beneficial in metallization of the polymers to enhance the adherence. However, for applications in the wearable device, damaged and deformed fiber surface are fatal problems to maintain the reliability of the electronic device. On the other hand, the surface of the Nylon 6,6 textile treated by the SCC was very similar to that of the untreated textile, and no defects were observed as shown in

Fig. 4. This result confirmed that the Nylon 6.6 fibers would not be damaged in the SCC. 
162 identified from the 3D images shown in Fig. 5. More details of the Ni-P coatings on the 163 fibers could be observed from the SEM images shown in Fig. 6. For the Ni-P coatings 164 deposited by the Conv-Cat followed by the Conv-ELP, the surface was not uniform and 165 rough, and some nodules and cracks were observed as shown in Fig. 6 (a). The rough 166 surface was suggested to be caused by the Conv-Cat and the hydrogen bubbles 167 generated during the electroless plating reactions provided in the following [20]:

- $\mathrm{Ni}^{2+}{ }_{\text {ads }}+\mathrm{H}_{2} \mathrm{PO}_{2}{ }_{\text {ads }}^{-}+\mathrm{H}_{2} \mathrm{O} \rightarrow \mathrm{Ni}^{0}+3 \mathrm{H}^{+}+\mathrm{HPO}_{3}{ }^{2-}$

- $\mathrm{H}_{2} \mathrm{PO}_{2}^{-}$ads $+\mathrm{H}_{2} \mathrm{O} \rightarrow \mathrm{HPO}_{3}^{2-}+\mathrm{H}^{+}+2 \mathrm{H}_{\mathrm{ads}}$

- $\mathrm{H}_{2} \mathrm{PO}_{2}{ }^{-}$ads $+\mathrm{H}_{\mathrm{ads}} \rightarrow \mathrm{P}^{0}+\mathrm{H}_{2} \mathrm{O}+\mathrm{OH}^{-}$

172 The hydrogen bubbles could adsorb on the surface to cause formation of the defects. As

173 shown Fig. 6 (b) and (c), smooth surface and no pin-holes were observed on the Ni-P

174 coating deposited by the SCC followed by the Conv-ELP or the ELP-SCE. However,

175 the Ni-P coating deposited by the SCC followed by the Conv-ELP had more peeled-off

176 parts when compared with the coating by the SCC followed by the ELP-SCE. The

177 peeled-off parts demonstrated that the adherence of the Ni-P coating by the Conv-ELP was low because of the insufficient anchoring effect of the metal coating on the fiber 
179 [21]. In contrast, the adherence of the Ni-P deposited by the ELP-SCE was high because

180 of the high anchoring effect given by impregnation of the Pd catalysts into the Nylon

1816,6 fibers.

182 The smooth surface of the Ni-P deposited by the ELP-SCE was suggested to be 183 caused by the sc- $\mathrm{CO}_{2}$ emulsified electrolyte [22]. The emulsified electrolyte contains a 184 continuous phase composed of the aqueous electrolyte and dispersed phases composed 185 of sc- $-\mathrm{CO}_{2}$ and the surfactant. Surface tension of $\mathrm{sc}-\mathrm{CO}_{2}$ is very low, and $\mathrm{CO}_{2}$ is nonpolar. Also, the dispersed phases would continuous bounce on the substrate surface during the electroless plating process, which then enhance removal of the adsorbed hydrogen gas bubbles from surface of the substrate to prevent generation of the defects $[23,24]$.

\subsection{Cross-sections of the metallized textile}

192

Cross-sectional SEM and EDS images of the Ni-P coating deposited on the Nylon deposited by the Conv-Cat followed by the Conv-ELP as indicated by the arrows in Fig. 
the Conv-ELP $(1 \mathrm{~min})$ was $7.03 \mu \mathrm{m}$. The coating thickness is thicker than the Ni-P SCC (120 min) followed by the ELP-SCE (60 min), which were 5.28 and $1.06 \mu \mathrm{m}$, respectively. In general, the Ni-P deposition rate was lower when either or both the SCC and the ELP-SCE were used.

The low deposition rate for the SCC when compared with the Conv-ELP is suggested to be a result of the difference in the crystallinity of the Pd catalyst after the catalyzation step. The Conv-Cat is expected to give Pd nanocrystallines on the Nylon 6,6 surface. On the other hand, the SCC could impregnate the Pd catalyst deep into the Nylon 6,6 to improve the adherence but the crystallinity of the $\mathrm{Pd}$ is poor. The Pd impregnated into the Nylon 6,6 is either Pd crystals having size in the atomic scale or Pd ions in the form of the Pd organo complex. The Pd organo complex would be reduced to metallic Pd after immersion in the electroless plating solution since there are reducing agents in the electroless plating solution. The poor crystallinity of the Pd catalyst by the SCC caused the low efficiency of the $\mathrm{Ni}$ reduction reaction and the low deposition rate. caused by a phenomenon often observed in applications of the emulsified electrolyte, which is named the "Periodic Plating Characteristic (PPC)". For the PPC, adsorption 
214 and desorption of the sc- $\mathrm{CO}_{2}$ dispersed phases from surface of the substrate could retard

215 the metal ion reduction reaction periodically [23]. However, the PPC might not be

216 enough to explain the low deposition rate in the ELP-SCE. Another explanation is

217 related to dissociation of $\mathrm{CO}_{2}$ into the aqueous electroless plating solution. The

218 dissociation of $\mathrm{CO}_{2}$ into the electroless plating solution would causes a decrease in the

$219 \mathrm{pH}$ as indicated in equation (5) $[25,26]$.

220

$$
\mathrm{CO}_{2}+\mathrm{H}_{2} \mathrm{O} \leftrightarrow \mathrm{H}_{2} \mathrm{CO}_{3} \leftrightarrow \mathrm{H}^{+}+\mathrm{HCO}_{3}^{-}
$$

221 When the $\mathrm{pH}$ of the electroless plating solution is low, it is expected to cause a

222 decrease in the film growth rate [27].

223 Also, the surface smoothening effect is suggested to a result of the PPC [24]. The

224 continuous adsorption and desorption of the sc- $\mathrm{CO}_{2}$ dispersed phases to a fine convex

225 part on the Ni-P coating can affect the nodule formation during the ELP-SCE and

226 eventually suppresses the growth of the nodules. 


\subsection{Effect of the deposition time and catalyzation time}

Fig. 8 shows the Ni-P coatings deposited by various lengths of the catalyzation and

232 plating times in the SCC and the ELP-SCE, respectively. The amount of the deposited

233 Ni-P was increased with an increased in either or both of the catalyzation time and the

234 plating time. The $\mathrm{Pd}$ organo complex could dissolve into the $\mathrm{sc}-\mathrm{CO}_{2}$ and then

235 impregnate into the Nylon 6,6 fibers. With a longer catalyzation time, the amount of the

236 Pd catalyst into the fibers would be increased and caused an increase in the amount of

237 the Ni-P deposited. The increase in the Ni-P coverage with an increase in the plating time during the ELP-SCE is expected since a longer plating time gives a higher amount of the reduced Ni-P. after the ELP-SCE was conducted as shown in Fig. 9. When 120 min of the SCC and 20 min of the ELP-SCE were used, nodule-like morphology was observed, and the nodule was confirmed to be mostly composed of $\mathrm{Ni}$ as shown in Fig. 9 (a). As the plating time was increased to $40 \mathrm{~min}$, an increase in size of the nodules was observed. Complete coverage of the Nylon 6,6 fibers with the Ni-P coatings was obtained when the plating time was increased to 60 min as shown in Fig. 9 (c). 

reactions proceed, the hemispherical particles gradually grow and connect to each other to form continuous coatings and eventually cover the entire surface.

\section{Conclusions} textile. When the Nylon 6.6 textiles were catalyzed in the $\mathrm{PdCl}_{2} / \mathrm{SnCl}_{2}$ solution, the

257 fibers were found to be damaged. The damage was suggested to be caused by the acid contained in the $\mathrm{PdCl}_{2} / \mathrm{SnCl}_{2}$ solution. On the other hand, the Nylon 6.6 textiles was not damaged when the SCC was applied. More uniform Ni-P coating on the surface of each Nylon 6,6 fiber was obtained when both the SCC and ELP-SCE were applied when compared with Ni-P coating deposited by the Conv-Cat and the Conv-ELP. As both the catalyzation time in the SCC and the plating time in the ELP-SCE were increased, the amount of Ni-P deposited on the textile was increased. These results demonstrated that 
264 the SCC and the ELP-SCE are both greatly promising processes for metallization of the 265 polymer textile.

\section{List of abbreviation}

268 Conv-Cat: conventional catalyzation

269 Conv-ELP: conventional electroless plating

270 EDS: energy dispersive X-ray spectrometry

271 ELP-SCE: electroless plating with supercritical $\mathrm{CO}_{2}$ emulsified electrolyte

272 OM: optical microscope

273 PEEK: polyether ether ketone

274 PPC: periodic plating characteristic

275 SCC: supercritical $\mathrm{CO}_{2}$ catalyzation

276 Sc- $\mathrm{CO}_{2}$ : supercritical $\mathrm{CO}_{2}$

277 SCE: supercritical $\mathrm{CO}_{2}$ emulsified electrolyte 
278 SEM: scanning electron microscope

279

280 Acknowledgments

281 This work has been supported by The Grant-in-Aid for Scientific Research (B) (JSPS

282 KAKENHI Grant Number 26282013) and CREST Project operated by the Japan

283 Science and Technology Agency (JST).

284

285

286

287

288

289

290

291

292 


\section{Reference}

294 [1] J. Brand, M. Kok, M. Koetse, M. Cauwe, R. Verplancke, F. Bossuyt, M. Jablonski,

295 J. Vanfleteren, Flexible and stretchable electronics for wearable health devices, 296 Solid-State Electronics, 113 (2015) 116-120.

297 [2] A. Affanni, G. Chiorboli, Design and characterization of a real-time, wearable,

298 endosomatic electrodermal system, Measurement, 75 (2015) 111-121.

299 [3] G. K. Lewis jr, M. D. Langer, C. R. Henderson jr, R Ortiz, Design and Evaluation of 300 a Wearable Self-Applied Therapeutic Ultrasound Device for Chronic Myofascial Pain, 301 Ultrasound in Medicine \& Biology, 39 (2013) 1429-1439.

302 [4] P. Sigmund, Mechanisms and theory of physical sputtering by particle impact, 303 Nuclear Instruments and Methods in Physics Research, B27 (1987) 1-20.

304 [5] K.L. Choy, Chemical vapour deposition of coatings, Progress in Materials Science, $30548(2003) 57-170$.

306 [6] V. M. Dubin, Y. S. Diamand, Selective and Blanket Electroless Copper Deposition 307 for Ultralarge Scale Integration, Journal of The Electrochemical Society, 144 (1997) 898-908. 
309 [7] J. P. Friedrich, E. H. Pryde, Supercritical CO2 extraction of lipid-bearing materials

310 and characterization of the products, Journal of the American Oil Chemists' Society, 61

311 (1984) 223-228.

312 [8] G.J.K. Bonthuys, C.E. Schwarz, A.J. Burger, J.H. Knoetze, Separation of alkanes

313 and alcohols with supercritical fluids. Part I: Phase equilibria and viability study, The

314 Journal of Supercritical Fluids, 57 (2011) 101-111.

315 [9] C.E. Schwarz, G.J.K. Bonthuys, R.F. van Schalkwyk, D.L. Laubscher, A.J. Burger,

316 J.H. Knoetze, Separation of alkanes and alcohols with supercritical fluids. Part II.

317 Influence of process parameters and size of operating range, The Journal of

318 Supercritical Fluids, 58 (2011) 352- 359.

319 [10] B. Subramanlam, M. A. McHugh, Reactions in Supercritical Fluids-A Review,

320 Industrial \& Engineering Chemistry Process Design and Development, 25 (1986) 1-12.

321 [11] J.M.Blackburn, D.P.Long, A.Cabanas, J.J. Watkins, Deposition of Conformal

322 Copper and Nickel Films from Supercritical Carbon Dioxide, Science, 294 (2001)

323 141-145. 
324 [12] E.Kondoh, J.Fukuda, Deposition kinetics and narrow-gap-filling in $\mathrm{Cu}$ thin film

325 growth from supercritical carbon dioxide fluids, Journal of Supercritical Fluids, 44

326 (2008) 466-474.

327 [13] M. Belmas, I. Tabata, K. Hisada, T. Hori, Supercritical fluid-assisted electroless

328 metal plating onto aramid films: The influence of thermal treatment, Journal of Applied

329 Polymer Science, 119 (2011) 2283-2291.

330 [14] T. Shimizu, Y. Ishimoto, T.F.M. Chang, H. Kinashi, T. Nagoshi, T. Sato, M. Sone,

$331 \mathrm{Cu}$ wiring into nano-scale holes by electrodeposition insupercritical carbon dioxide

332 emulsified electrolytewith a continuous-flow reaction system, The Journal of

333 Supercritical Fluids, 90 (2014) 60-64.

334 [15] S. Tengsuwan, M. Ohshima, Supercritical carbon dioxide-assisted electroless

335 nickel plating on polypropylene-The effect of copolymer blend morphology on

336 metal-polymer adhesion, Journal of Supercritical Fluids, 85 (2014) 123- 134.

337 [16] B.H. Woo, M. Sone, A. Shibata, C. Ishiyama, S. Edo, M. Tokita, J. Watanabe, Y. 338 Higo, Impregnation of $\mathrm{Ni}-\mathrm{P}$ metal into polymer substrate via catalyzation in $\mathrm{Sc}-\mathrm{CO} 2$ 339 and electroless plating in Sc-CO2 emulsion, Surface \& Coatings Technology 204 (2010) 1785-1792. 
341 [17] H. Qin, Q. Su, S. Zhanga, B. Zhao, M. Yang, Thermal stability and flammability of

342 polyamide 66/montmorillonite nanocomposites, Polymer, 44 (2003) 7533-7538.

343 [18] B. Suresha, B.N. Ravi Kumar, M. Venkataramareddy, T. Jayaraju, Role of 344 micro/nano fillers on mechanical and tribological properties of 345 polyamide66/polypropylene composites, Materials and Design, 31 (2010) 1993-2000.

346 [19] R.M. Mcdonogh, C.J.D. Fell, A.G. Fane, Characteristics of membranes formed by

347 acid dissolution of polyamides, Journal of Membrane Science, 31 (1987) 321-336.

348 [20] A. Małecki, A. M. Ilnicka, Electroless nickel plating from acid bath, Surface and 349 Coatings Technology, 123 (2000) 72-77.

350 [21] U. K. Fatema, Y. Gotoh, A new electroless Ni plating procedure of iodine-treated 351 aramid fiber, Journal of Coatings Technology and Research, 10 (2013) 415-425.

352 [22] H. Yoshida, M. Sone, A. Mizushima, K. Abe, X. T. Tao, S. Ichihara, S. Miyata, 353 Electroplating of Nanostructured Nickel in Emulsion of Supercritical Carbon Dioxide in 354 Electrolyte Solution, Chemistry Letters, 10 (2002) 1086-1087. 
355 [23] T.F.M. Chang, M. Sone, A. Shibata, C. Ishiyama, Y. Higo, Bright nickel film 356 deposited by supercritical carbon dioxide emulsion using additive-free Watts bath, 357 Electrochimica Acta, 55 (2010) 6469-6475.

358 [24] T.F.M. Chang, Masato Sone, Function and mechanism of supercritical carbon 359 dioxide emulsified electrolyte in nickel electroplating reaction, Surface \& Coatings 360 Technology, 205 (2011) 3890-3899.

361 [25] H. Uchiyama, M. Sone, A. Shibata, Y. Higo, Effects of $\mathrm{CO}_{2}$ on Ni-P Electroless 362 Plating in an Emulsion of Supercritical $\mathrm{CO}_{2}$, Journal of The Electrochemical Society, 363157 (2010) D550-D552.

364 [26] H. Uchiyama, T. Endo, M. Sone, Direct Observation of Nodule Growth on 365 Electroless Ni-P Deposition in Supercritical CO2 Emulsion, Journal of The 366 Electrochemical Society, 159 (2012) D114-D118.

367 [27] G. O. Malloy, J. B. Hajdu, Electroless Plating: Fundamentals and Applications, 368 American Electroplaters and Surface Finishers Society, Orlando, FL (1990). 
372 Figure 1. (a) $\mathrm{CO} 2$ gas tank, (b) $\mathrm{CO}_{2}$ liquidization unit, (c) Liquidizatrion unit, (d)

373 High pressure pump, (e) Thermal bath, (f) Reaction-cell (PEEK-lined SUS316L), (g)

374 Textile, (h) Cross stirrer, (i) Back pressure regulator, (j) Trap

375

376 Figure 2, 3D images of the catalyzed textile in OM: (a) Untreated Nylon 6,6 textile, (b)

377 Catalyzed Nylon 6,6 textile by Conv-Cat for 10 s, (c) Catalyzed Nylon 6,6 textile by 378 SCC for $120 \mathrm{~min}$.

380 Figure 3, OM images of the surfaces: Untreated Nylon 6,6 textile in (a) low and (b) high magnification, and catalyzed Nylon 6,6 textile by the Conv-Cat for $10 \mathrm{~s}$ in (c) low and (d) high magnification. The white rectangular dash mark shows part of the damaged fiber and the black circular dash marks shows the white particulates.

Figure 4, OM images of the surfaces: Nylon 6,6 textile treated by the SCC in (a) low and (b) high magnification. 
388 Figure 5, 3D images of the Ni-P coated textiles observed by the OM: (a) The Ni-P 389 coated textile by the Conv-Cat and the Conv-ELP, (b) the Ni-P coated textile by the 390 SCC and the Conv-ELP, and (c) the Ni-P coated textile by the SCC and the ELP-SCE.

392 Figure 6, SEM images of the Ni-P coated fibers: (a) The Conv-Cat for $10 \mathrm{~s}$ and the 393 Conv-ELP for $1 \mathrm{~min}$, (b) the SCC for $120 \mathrm{~min}$ and the Conv-ELP for $20 \mathrm{~min}$, and (c) 394 the SCC for $120 \mathrm{~min}$ and the ELP-SCE for $60 \mathrm{~min}$.

396 Figure 7, Cross-sectional SEM and EDS images of the Ni-P coatings on the fiber: (a) 397 the Conv-Cat for $10 \mathrm{~s}$ and the Conv-ELP for $1 \mathrm{~min}$, (b) the SCC for $120 \mathrm{~min}$ and the 398 Conv-ELP for $20 \mathrm{~min}$, (c) the SCC for $120 \mathrm{~min}$ and the ELP-SCE for $60 \mathrm{~min}$. Red parts 399 are signals of Ni detected by the EDS. The white arrows indicate the peeled parts in (a).

400

401

402 
404 Figure 8, Surfaces of the Ni-P coatings with various time in the SCC and the ELP-SCE:

405 (a) SCC for $20 \mathrm{~min}$ and ELP-SCE for $20 \mathrm{~min}$, (b) SCC for $20 \mathrm{~min}$ and ELP-SCE for 40 $406 \mathrm{~min}$, (c) SCC for $20 \mathrm{~min}$ and ELP-SCE for $60 \mathrm{~min}$, (d) SCC for $60 \mathrm{~min}$ and ELP-SCE 407 for $20 \mathrm{~min}$, (e) SCC for $60 \mathrm{~min}$ and ELP-SCE for $40 \mathrm{~min}$, (f) SCC for $60 \mathrm{~min}$ and 408 ELP-SCE for $60 \mathrm{~min}$, (g) SCC for $120 \mathrm{~min}$ and ELP-SCE for $20 \mathrm{~min}$, (h) SCC for 120 $409 \mathrm{~min}$ and ELP-SCE for $40 \mathrm{~min}$, (i) SCC for $120 \mathrm{~min}$ and ELP-SCE for $60 \mathrm{~min}$.

411 Figure.9, SEM and EDS mapping images of the Ni-P deposited on the fibers by (a) the 412 SCC for $120 \mathrm{~min}$ and the ELP-SCE for $20 \mathrm{~min}$, (b) the SCC for $120 \mathrm{~min}$ and the 413 ELP-SCE for $40 \mathrm{~min}$, and (c) the SCC for $120 \mathrm{~min}$ and the ELP-SCE for $60 \mathrm{~min}$. Red 414 parts are Ni detected by EDS. 


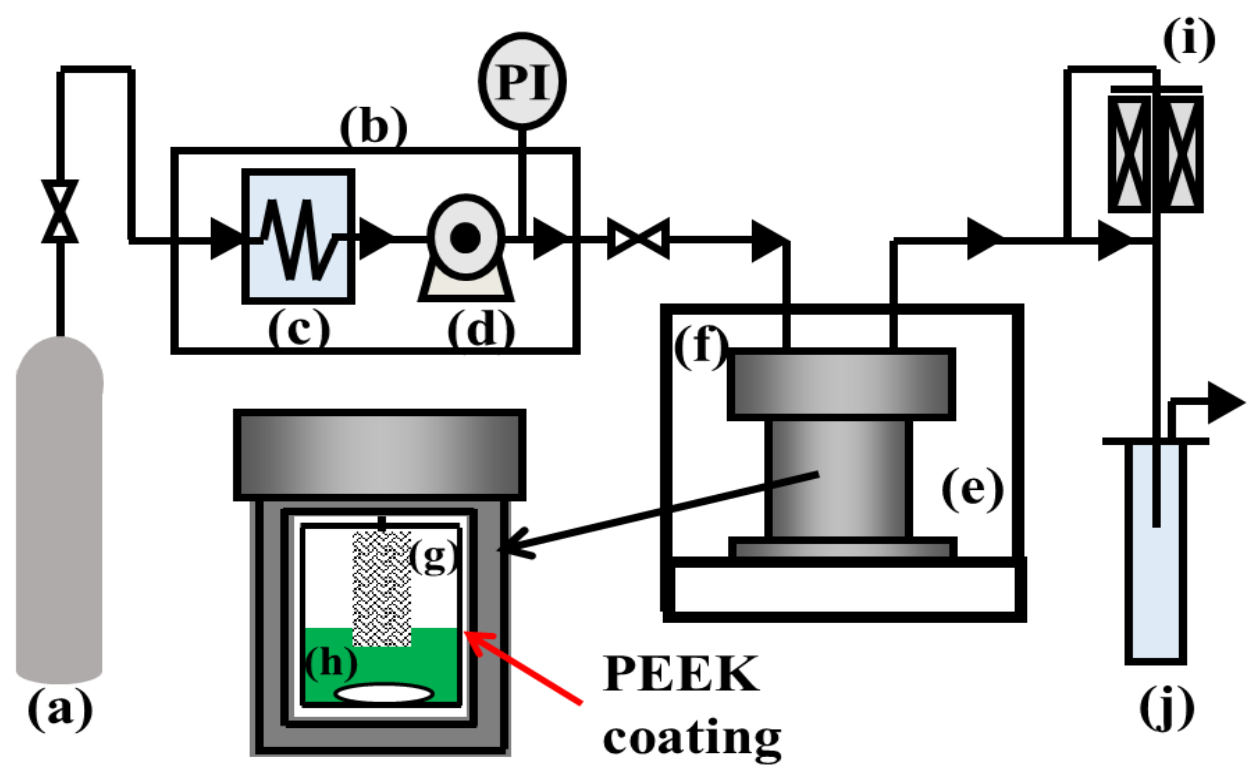

Figure 1, (a) $\mathrm{CO}_{2}$ gas tank, (b) $\mathrm{CO}_{2}$ liquidization unit, (c) Liquidizatrion unit, (d) High pressure pump, (e) Thermal bath, (f) Reaction-cell (PEEK-lined SUS316L), (g) Textiles, (h) Cross stirrer, (i) Back pressure regulator, (j) Trap. 


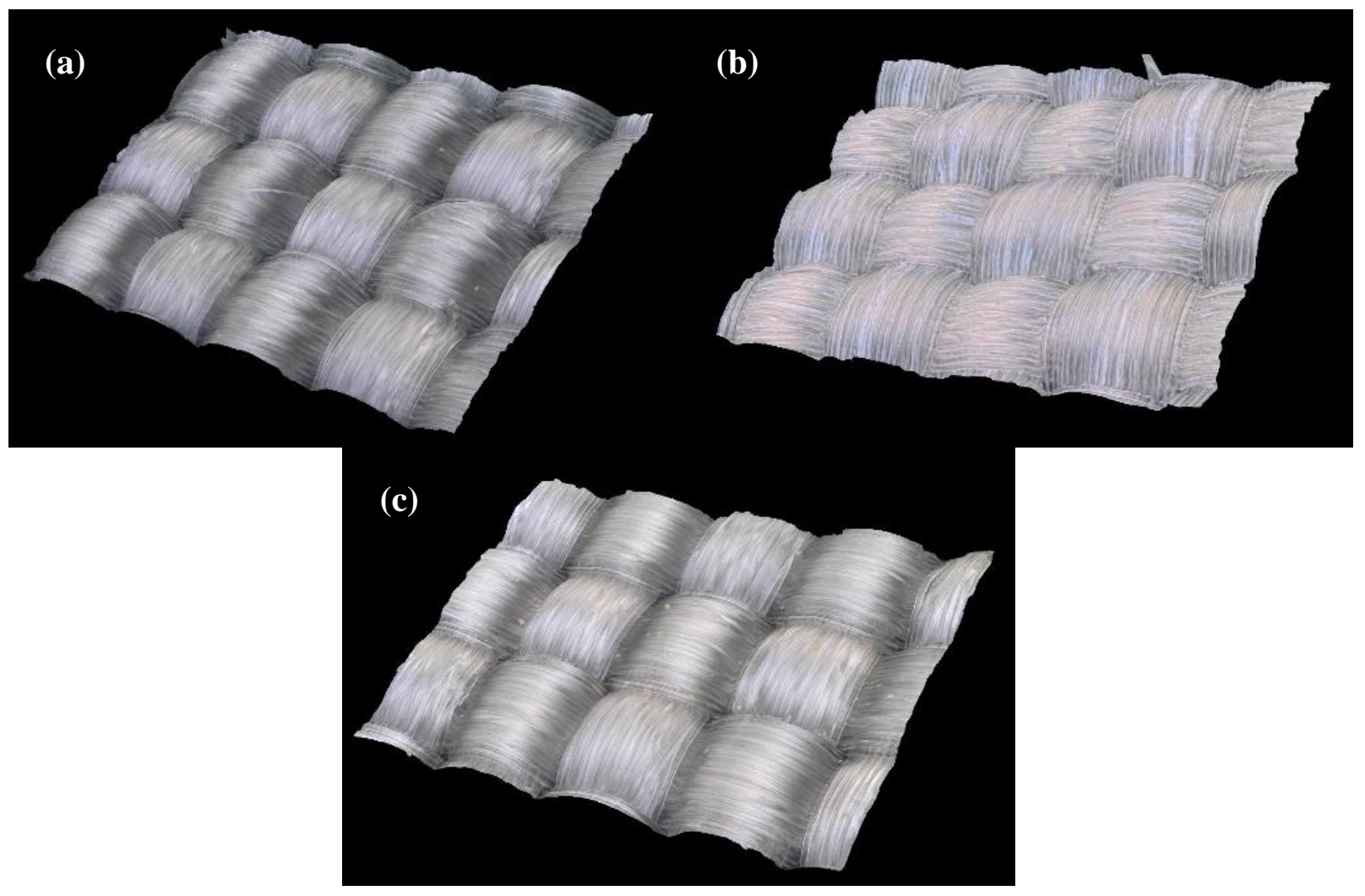

Figure 2, 3D images of the textiles observed by the OM: (a) Untreated Nylon 6,6 textiles, (b) Catalyzed Nylon 6,6 textiles by the Conv-Cat for $10 \mathrm{~s}$, and (c) Catalyzed Nylon 6,6 textiles by the SCC for $120 \mathrm{~min}$. 


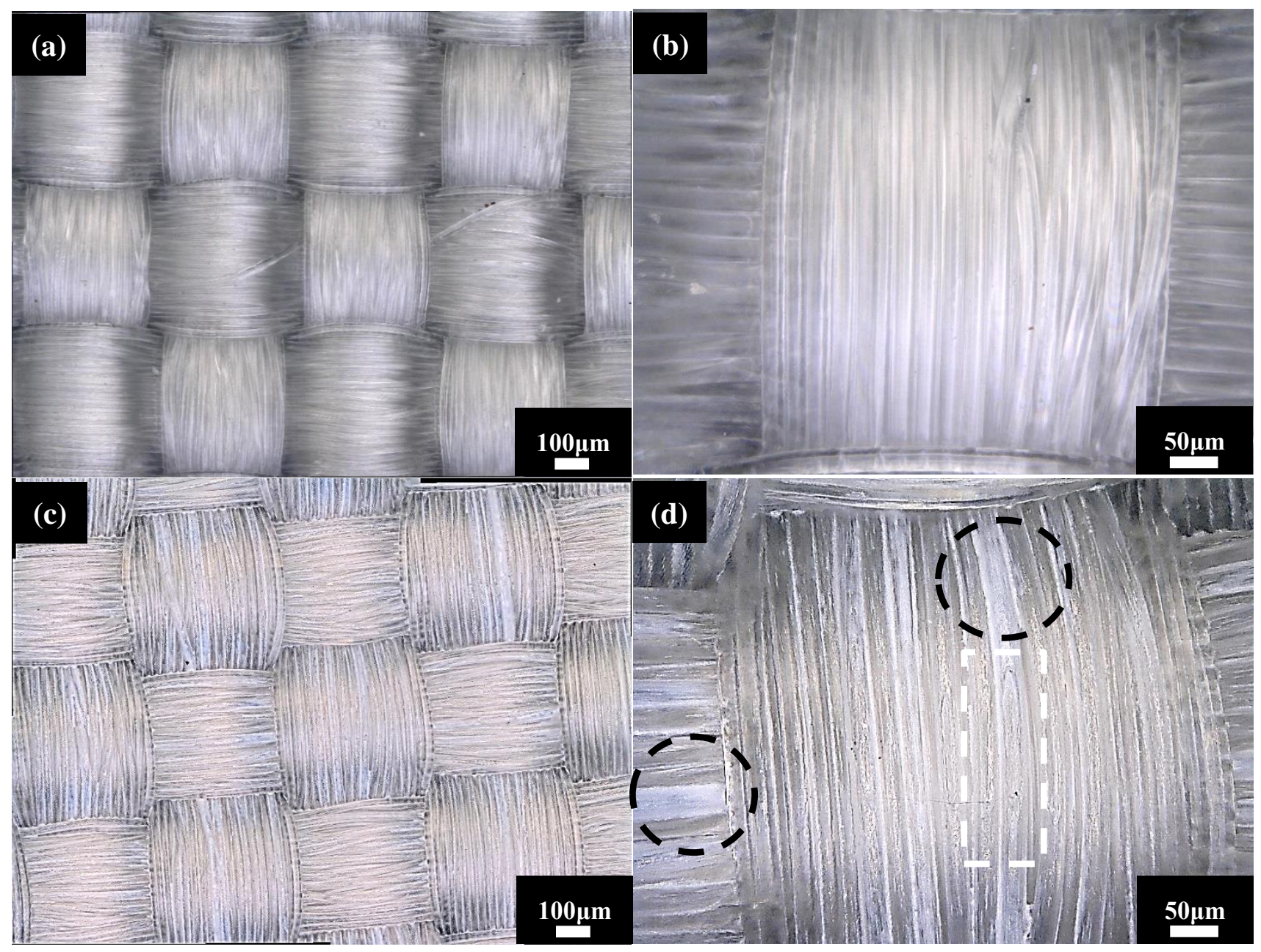

Figure 3, OM images of the surfaces: Untreated Nylon 6,6 textiles in (a) low and (b) high magnification, and catalyzed Nylon 6,6 textiles by the Conv-Cat for $10 \mathrm{~s}$ in (c) low and (d) high magnification. The white rectangular dash mark shows part of the damaged fiber and the black circular dash marks shows the white particulates. 


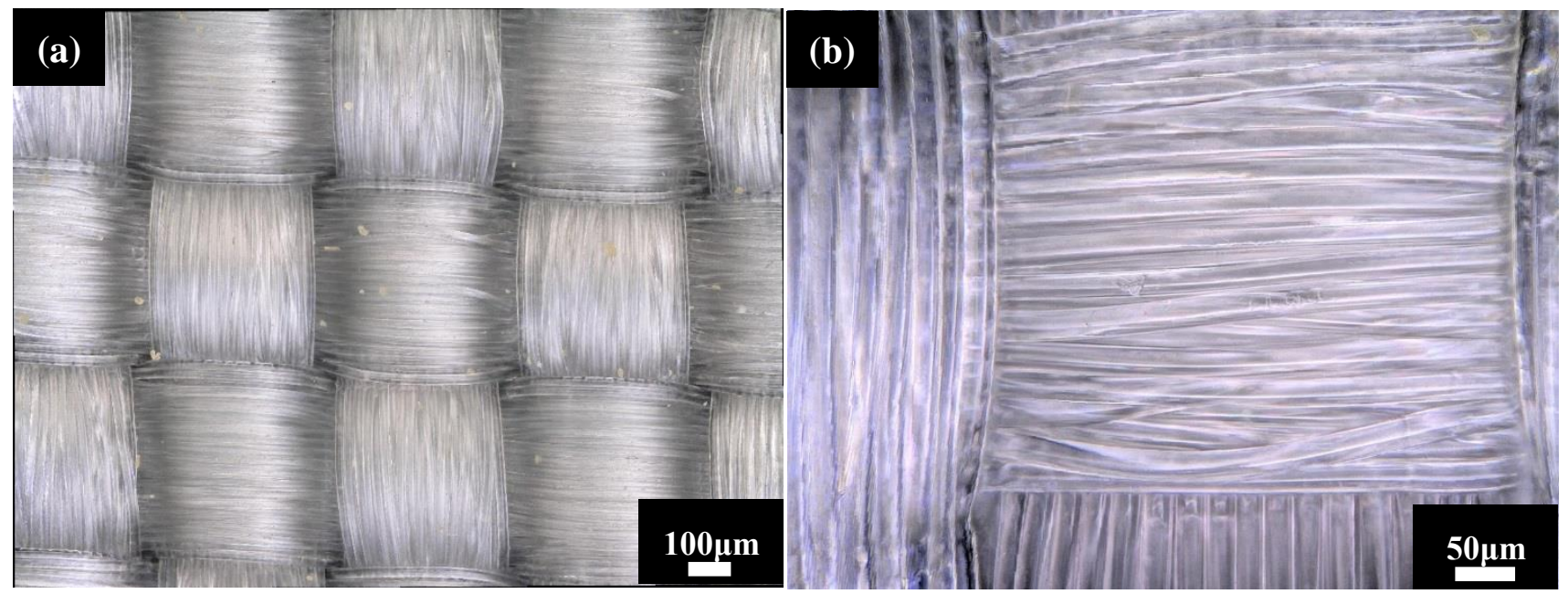

Figure 4, OM images of the surfaces: Nylon 6,6 textiles treated by the SCC in (a) low and (b) high magnification. 


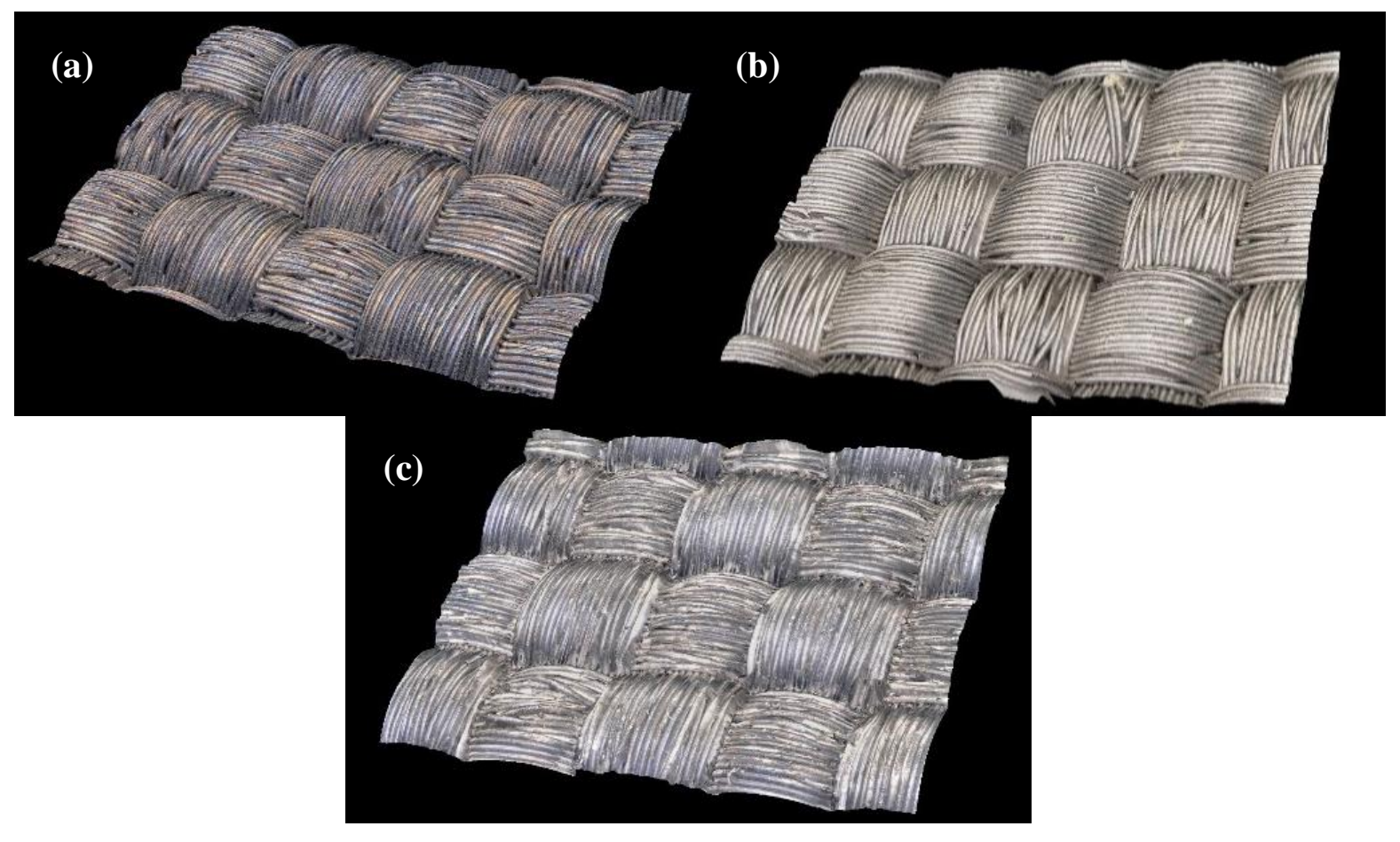

Figure 5, 3D images of the Ni-P coated textiles observed by the OM: (a) The Ni-P coated textiles by the Conv-Cat and the Conv-ELP, (b) the Ni-P coated textiles by the SCC and the Conv-ELP, and (c) the Ni-P coated textiles by the SCC and the ELP-SCE. 


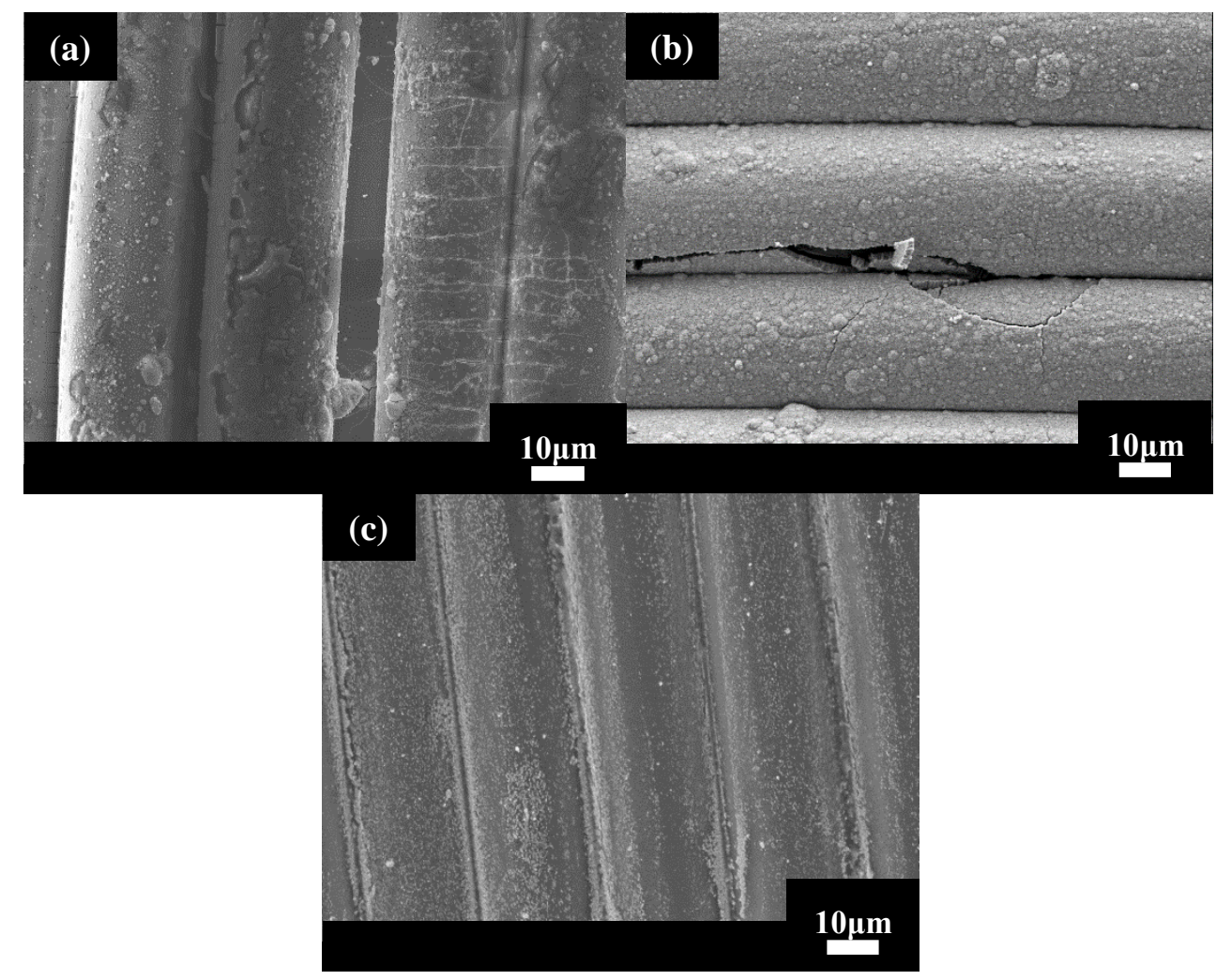

Figure 6, SEM images of the Ni-P coated fibers: (a) The Conv-Cat for $10 \mathrm{~s}$ and the Conv-ELP for $1 \mathrm{~min}$, (b) the SCC for $120 \mathrm{~min}$ and the Conv-ELP for 20 min, and (c) the SCC for $120 \mathrm{~min}$ and the ELP-SCE for $60 \mathrm{~min}$. 

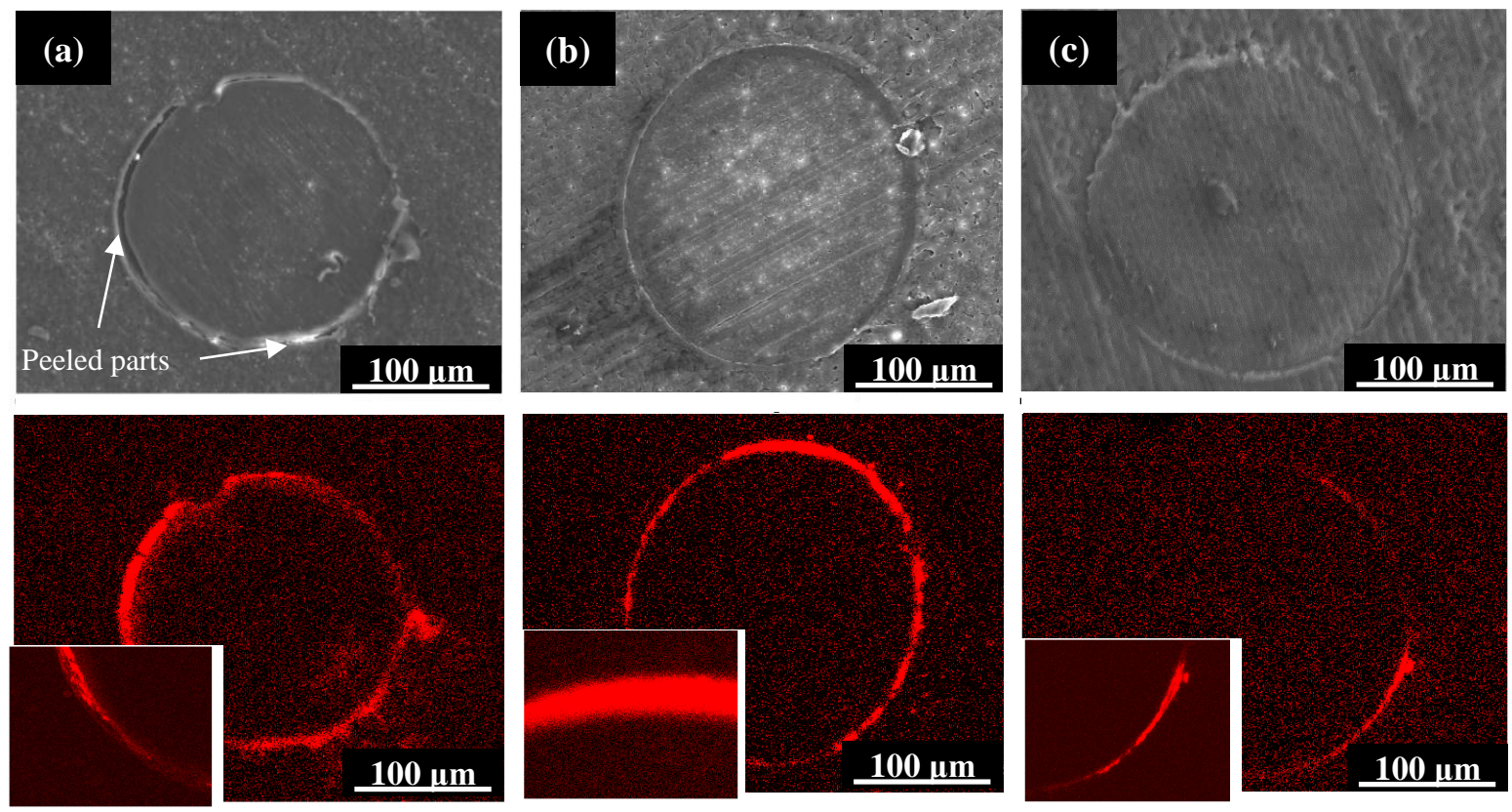

Figure 7, Cross-sectional SEM and EDS images of the Ni-P coatings on the fiber: (a) the Conv-Cat for $10 \mathrm{~s}$ and the Conv-ELP for $1 \mathrm{~min}$, (b) the SCC for $120 \mathrm{~min}$ and the Conv-ELP for $20 \mathrm{~min}$, (c) the SCC for $120 \mathrm{~min}$ and the ELPSCE for $60 \mathrm{~min}$. Red parts are signals of Ni detected by the EDS. The white arrows indicate the peeled parts in (a). 

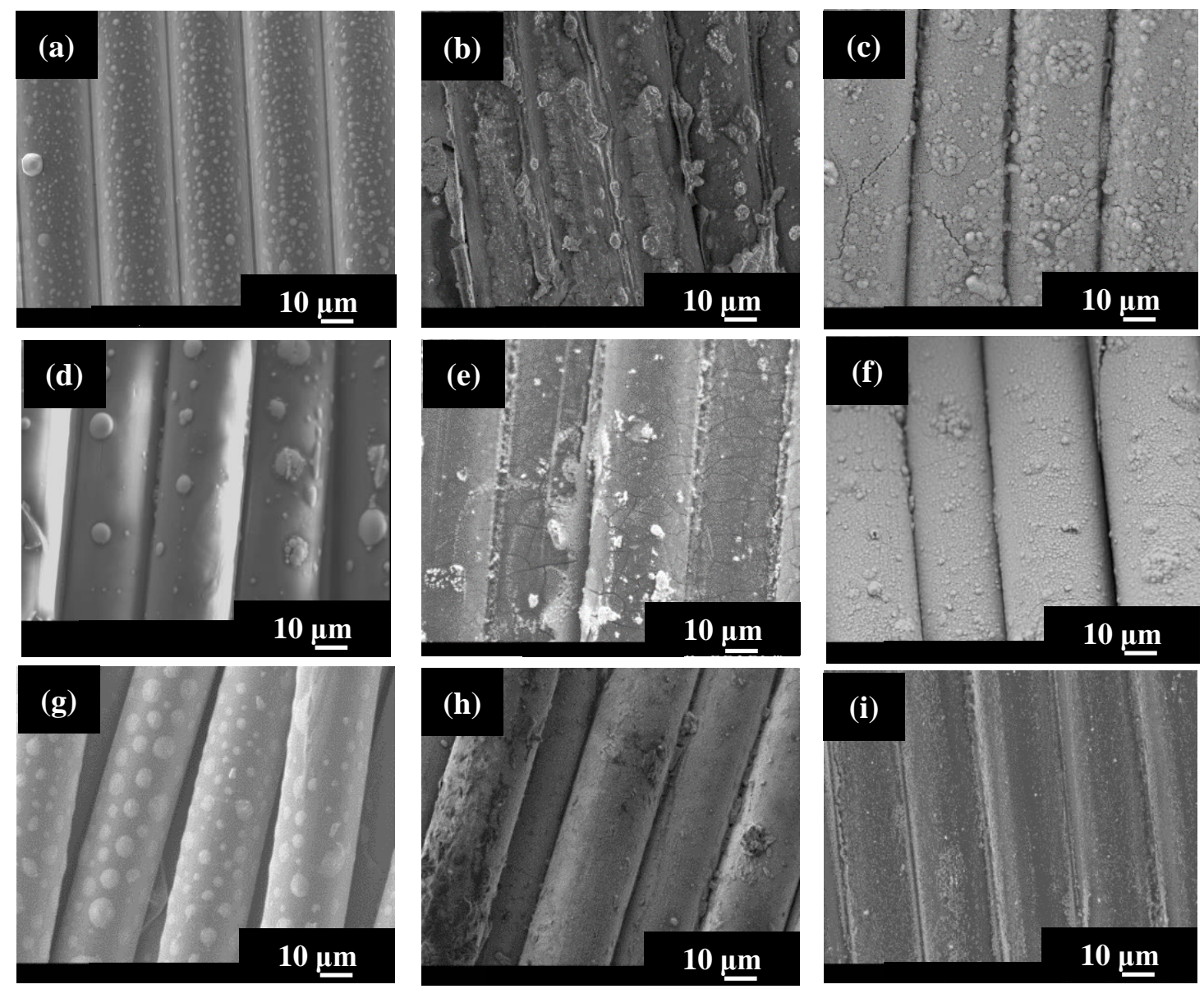

Figure 8, Surfaces of the Ni-P coatings with various time in the SCC and the ELP-SCE: (a) SCC for $20 \mathrm{~min}$ and ELP-SCE for $20 \mathrm{~min}$, (b) SCC for $20 \mathrm{~min}$ and ELP-SCE for $40 \mathrm{~min}$, , (c) SCC for $20 \mathrm{~min}$ and ELP-SCE for $60 \mathrm{~min}$, (d) SCC for $60 \mathrm{~min}$ and ELP-SCE for $20 \mathrm{~min}$, (e) SCC for $60 \mathrm{~min}$ and ELP-SCE for $40 \mathrm{~min}$, (f) SCC for $60 \mathrm{~min}$ and ELP-SCE for $60 \mathrm{~min}$, (g) SCC for $120 \mathrm{~min}$ and ELP-SCE for $20 \mathrm{~min}$, (h) SCC for $120 \mathrm{~min}$ and ELP-SCE for $40 \mathrm{~min}$, (i) SCC for $120 \mathrm{~min}$ and ELP-SCE for $60 \mathrm{~min}$. 


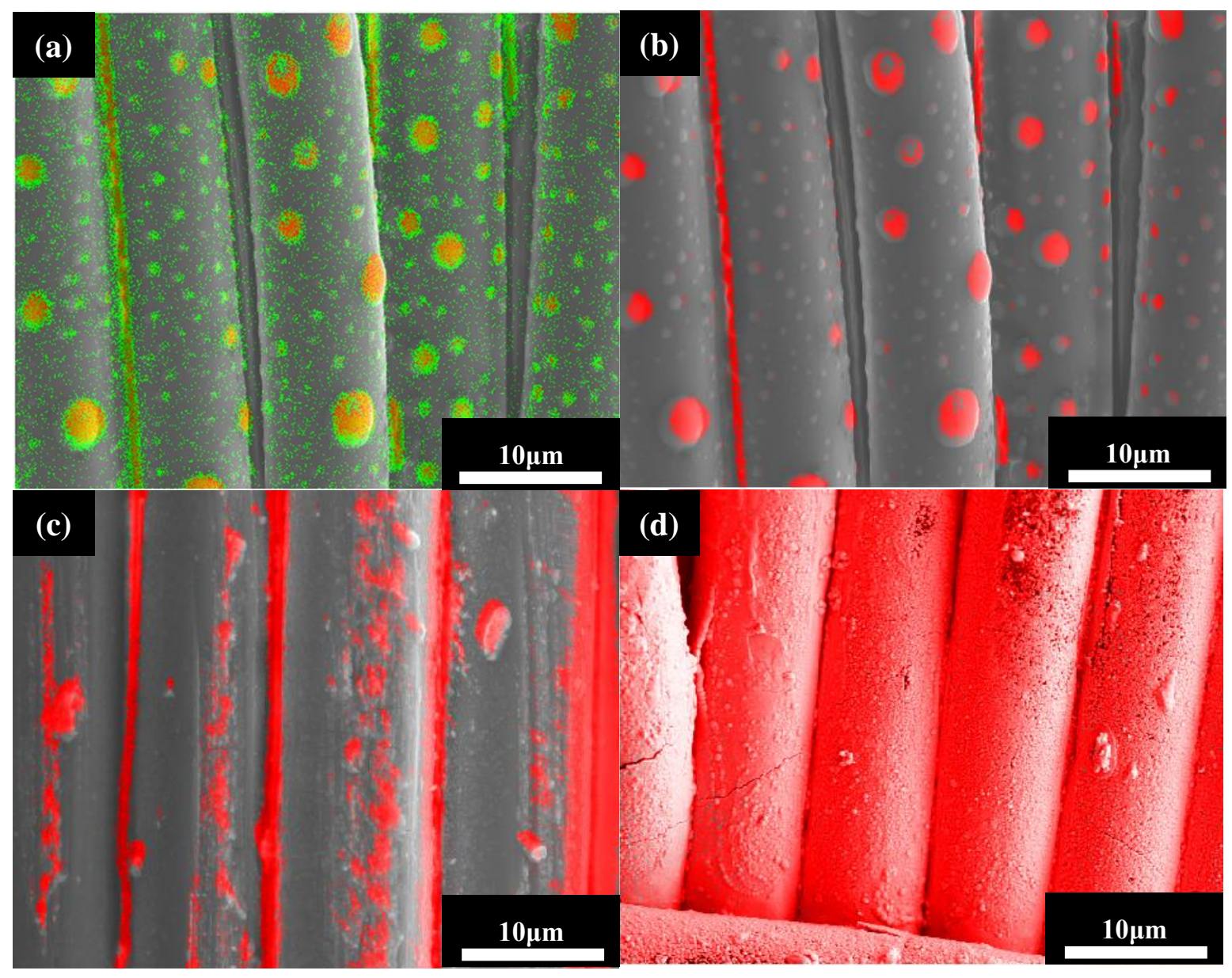

Figure 9, SEM and EDS mapping images of the Ni-P deposited on the fibers by (a) and (b) the SCC for $120 \mathrm{~min}$ and the ELP-SCE for $20 \mathrm{~min}$, (c) the SCC for $120 \mathrm{~min}$ and the ELP-SCE for $40 \mathrm{~min}$, and (d) the SCC for $120 \mathrm{~min}$ and the ELP-SCE for 60 min. Red parts are the Ni content and green parts are the $\mathrm{P}$ content detected by EDS. 


\section{$\underline{\text { Supercritical } \mathrm{CO}_{2} \text { Catalyzation (SCC) }}$}

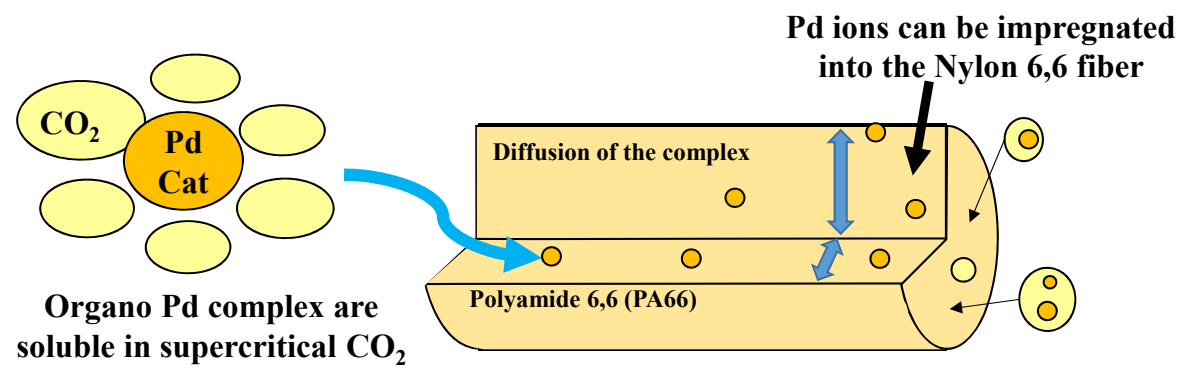

\section{$\left(\mathrm{sc}^{\left.-\mathrm{CO}_{2}\right)}\right.$}

Electroless plating with sc-CO $\mathrm{CO}_{2}$ emulsified electrolyte (ELP-SCE)

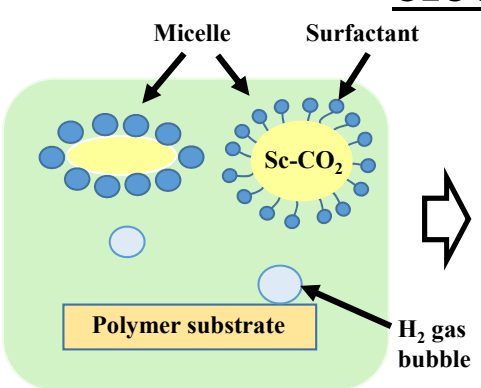

Emulsified electrolyte

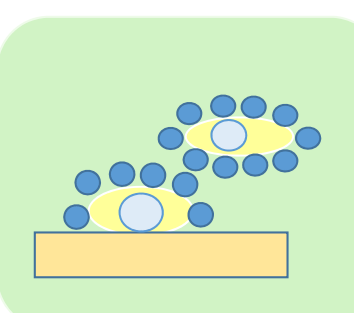

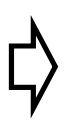

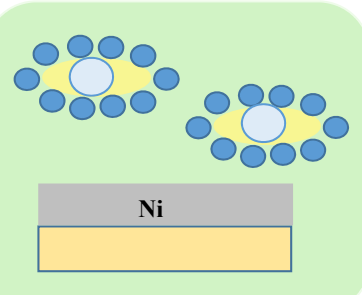

Micelle absorb $\mathrm{H}_{2}$ bubble

Defect-free Ni-P film

\section{Ni-P coated Nylon 6,6 textile}

Conventional catalyzation and SCC and conventional electroless plating electroless plating

SCC and ELP-SCE
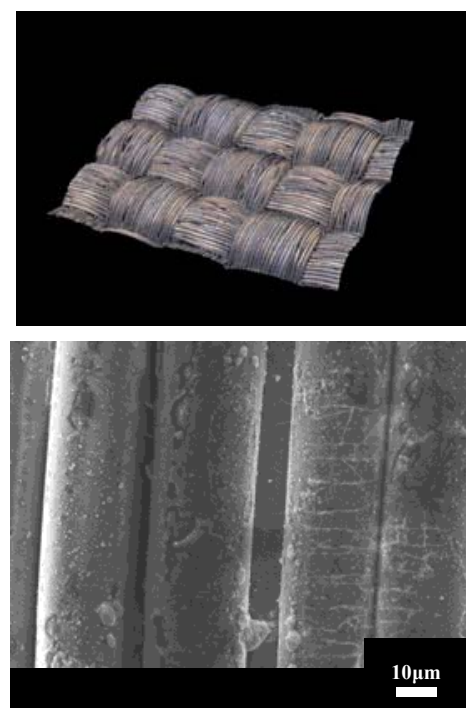

Many cracks

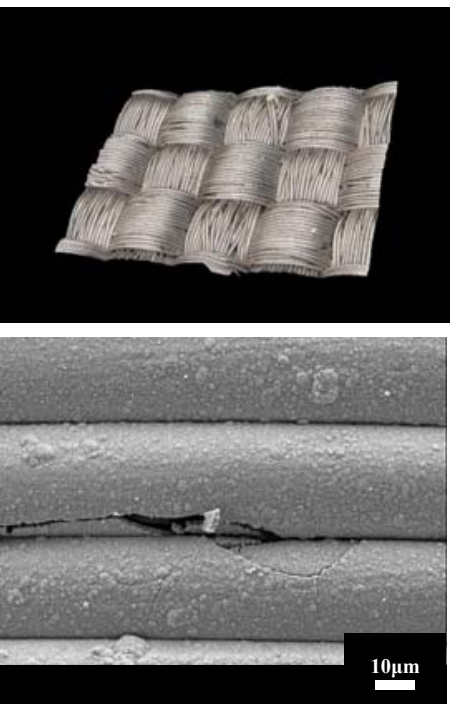

Some cracks

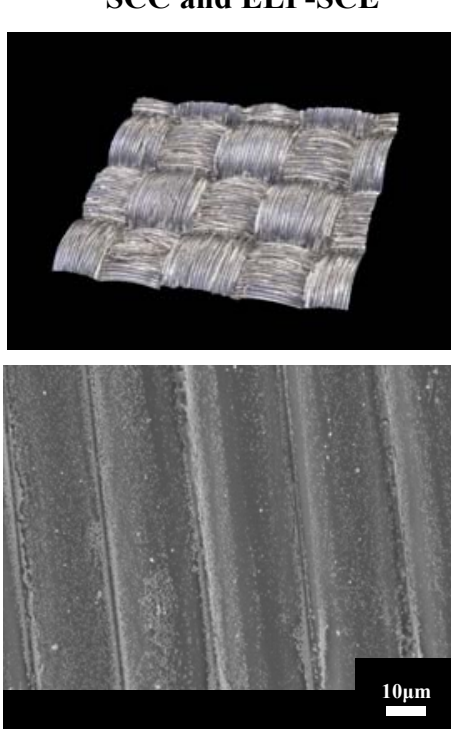

Uniform Ni-P coting 